\section{Clinical, histological and ultrastructural studies of the posterior hyaloid membrane}

\author{
Abstract \\ Aims To investigate the histological, \\ immunohistochemical and ultrastructural \\ features of the posterior hyaloid membrane \\ (PHM) in its naturally separated state in \\ patients without previous surgery and slit- \\ lamp documentation of antemortem posterior \\ vitreous detachment (PVD). \\ Methods A prospective study was \\ commenced in 1992 to recruit patients with \\ physiological PVD from an unselected group \\ of general medical inpatients and ascertain \\ the prevalence of PVD. Postmortem \\ specimens subsequently available were \\ studied to analyse the clinicopathological \\ correlation and processed using standard \\ techniques for histology, \\ immunohistochemistry and electron \\ microscopy.
}

Results Eighty-five patients were examined with ages ranging from 68 to 98 yrs (mean 83.4 yrs). The posterior hyaloid membrane had clearly separated from the retina in $66 \%$ of eyes. Twenty-nine eyes from 15 patients were subsequently studied pathologically. The posterior hyaloid membrane exhibited a uniform cellular component, most densely populated around the Weiss' ring. The cells were characterised by oval or round nuclei, indistinct cytoplasm and were only seen within, or abutting, the weakly eosinophilic posterior hyaloid membrane. The posterior aspect of the posterior hyaloid membrane showed a convoluted appearance staining lightly with haematoxylin and eosin. The detached posterior hyaloid membrane exhibited focal positivity for GFAP and type IV collagen. Electron microscopy demonstrates both fibres and basement membrane associated with the cellular component including hemi-desmosome attachment plaques between the cells and basement membrane.
MP Snead ${ }^{1}$, DRJ Snead ${ }^{2}$, AJ Richards ${ }^{3}$, JB Harrison ${ }^{3}$, AV Poulson ${ }^{1}$, AHC Morris ${ }^{1}$, RM Sheard ${ }^{1}$ and JD Scott ${ }^{1}$

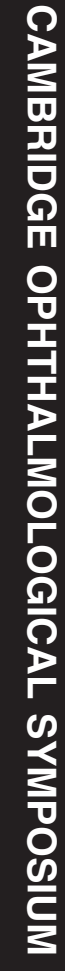

Conclusions This study illustrates some of the structural differences between the posterior hyaloid membrane and the cortical vitreous gel it envelopes and demonstrates the presence of cells intimately associated with the posterior hyaloid membrane in its naturally separated state. We propose the cellular population integral to the PHM to be designated as laminocytes in order to emphasise their type IV collagen/basement membrane association and planar array within the membrane which separates at posterior vitreous detachment.

Eye (2002) 16, 447-453. doi:10.1038/

sj.eye. 6700198

Keywords: posterior hyaloid membrane; posterior vitreous detachment; vitreous

\section{Introduction}

Posterior vitreous detachment (PVD) is characterised by a distinct membrane on the posterior aspect of the detached gel. Surgically this membrane can be extremely tough, and it has a characteristically crinkled glossy surface when viewed by specular slit-lamp biomicroscopy (Figure 1). Clinical observation suggests a structure quite different from the relatively loose cortical gel it envelopes, and its separation from the retina can even occur years after the vitreous body has been removed (Figure 2).

Much of the initial pathological research on PVD and the vitreoretinal interface was carried out on postmortem eyes and the contributions of Zimmerman and Straatsma, Gärtner, Foos, Heegaard and Seebag ${ }^{1-7}$ introduced new avenues for research on the vitreoretinal interface. Substantial variations individually and geographically in the structure known as the Inner Limiting
${ }^{1}$ Vitreoretinal Service Box 41

Addenbrooke's NHS Trust Hills Road

Cambridge, UK

${ }^{2}$ Department of Histopathology University Hospitals of Coventry and Warwickshire Clifford Bridge Road

Coventry, UK

${ }^{3}$ Department of Pathology Tennis Court Road Cambridge, UK

Correspondence: MP Snead MD FRCS FRCOphth

Vitreoretinal Service

Box 41

Addenbrooke's NHS Trust Hills Road

Cambridge CB2 2QQ, UK

Tel: 4401223216701

Fax: 4401223217968

E-mail:mps34@cam.ac.uk 


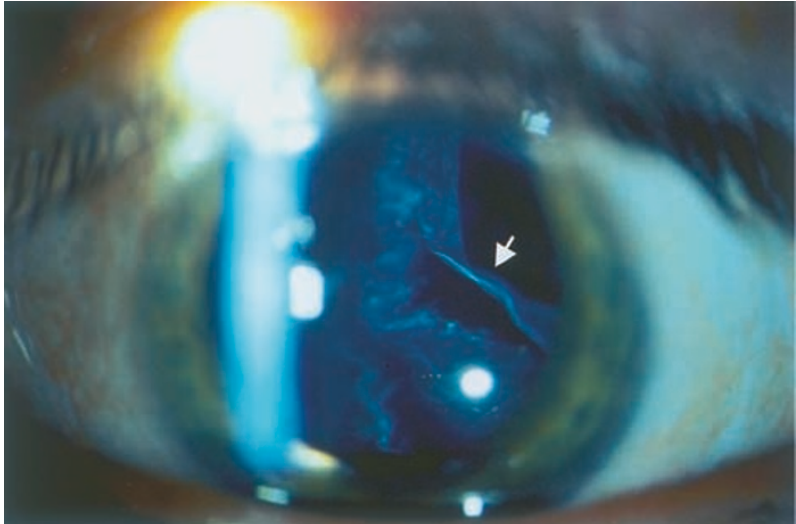

Figure 1 Detached posterior hyaloid membrane. The membrane is identified as a continuous mobile sheet which on slitlamp biomicroscopy exhibits a glossy, crinkled surface (arrow). Note contrast with the neighbouring cortical gel.

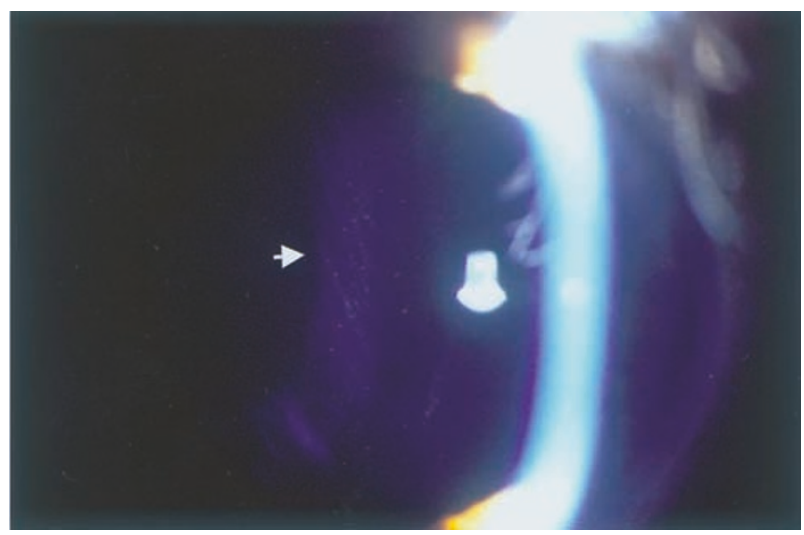

Figure 2 Detached posterior hyaloid membrane in a patient who has undergone previous pars plana vitrectomy for removal of an introcular foreign body. The cortical gel normally abutting the membrane is absent.

Membrane of the retina were found ${ }^{4,6}$ and that although the inner limiting membrane (ILM) increases in thickness throughout life, ${ }^{4,6}$ substantial variations in ILM thickness exist even between eyes of the same patient. ${ }^{6}$

Research to date has been limited by the paucity of antemortem clinical correlation so that exclusion of postmortem artefactual gel separation has been difficult. Alternatively, some studies have attempted to simulate postmortem gel separation by sequential removal of the ocular coats to reveal the main mass of the vitreous body. For these reasons much of the focus of previous research has concentrated on the ILM and vitreoretinal interface in its attached state. There is a lack of a good animal model and a natural bias towards investigation of pathology generally associated with retinal detachment and vitreoretinal pathology, especially lattice, proliferative vitreoretinopathy and epiretinal membranes.

We wished to address these areas by focusing our investigation on the vitreous and PHM in its naturally separated state and exclusively in patients without other posterior segment pathology or previous surgery.

\section{Patient recruitment and clinical studies}

Patients with physiological PVD were studied from an unselected group of general medical inpatients as previously reported. ${ }^{8,9}$ In brief, this ongoing prospective study was commenced 10 years ago with prior ethical approval (LREC DEC92/02). Inpatients were recruited from general medical wards with informed written consent to assess the prevalence of posterior vitreous detachment. A full past ocular and medical history was recorded. The protocol excluded patients with a history of familial eye disease or previous intraocular surgery (excluding uncomplicated cataract surgery). Vitreous detachment was defined as a clearly visible continuous sheet of posterior hyaloid membrane with or without an associated Weiss ring (Figure 1). The continuity of the separated membrane was inspected in detail since the presence of a Weiss ring alone was considered insufficient evidence alone for complete PVD. This is because a 'rhexis-like' defect in the posterior membrane adjacent to the Weiss ring can occur with incomplete gel separation from the retina. Alternatively, the Weiss ring can be disrupted at the time of separation. Eyes without media of sufficient clarity to make this judgement were not recruited to the study.

The hospital records of recruited patients were flagged. In the event of the patient's death, the relatives were approached for written informed consent for organ donation for research purposes. If consent was granted, specimens for light microscopy were enucleated and fixed in 10\% buffered formal saline.

In the air suspension technique described by Foos, ${ }^{3}$ a longitudinal section of the globe was made and the eye rotated to assess attachment or detachment of the gel from the retina. In this study, the patient's antemortem PVD status was known so the approach was modified in an attempt to preserve the integrity of the PHM. The specimens were processed in one of two ways. In the first group the globes were sectioned in parasagittal planes giving central, temporal and nasal callotes. The callotes were processed on an extended cycle as previously described ${ }^{8}$ and embedded in oversized paraffin wax blocks. Sections were cut at a number of levels, including through the optic disc and stained with haematoxylin and eosin and alcian blue, 


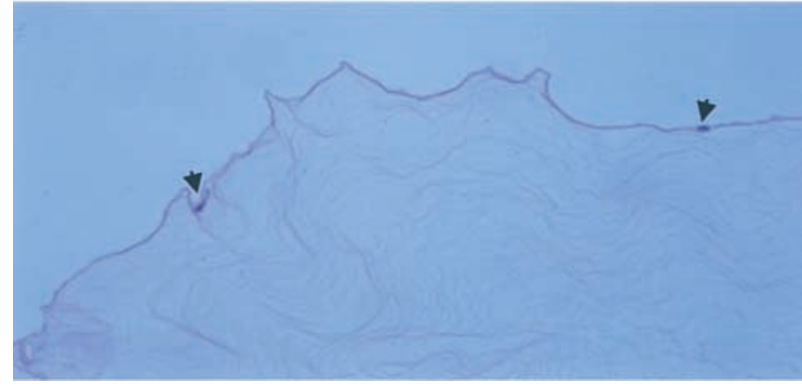

Figure 3 Detached posterior hyaloid membrane. Histological preparation from a patient with uncomplicated PVD. The laminocyte population is sparse in the peripheral membrane (arrows) H\&E $\times 200$ (compare with Figure 5).

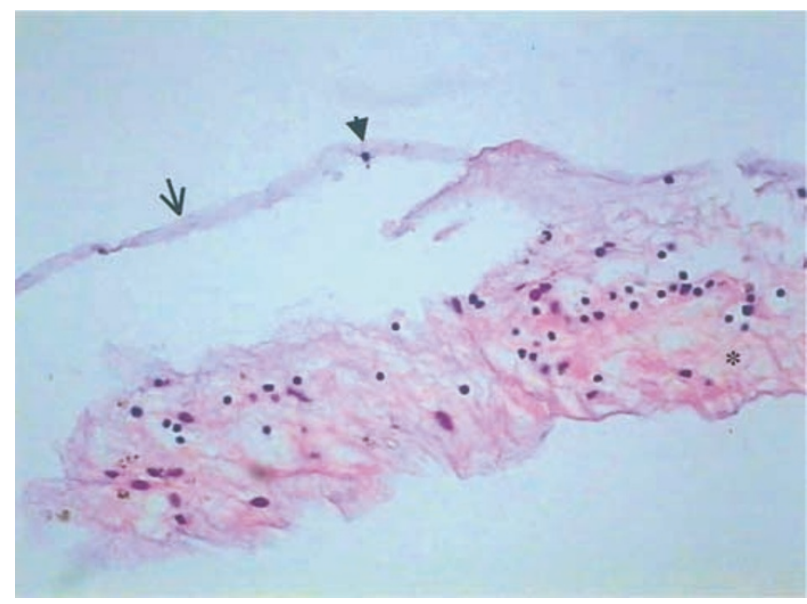

Figure 4 Detached posterior hyaloid membrane in a patient with an untreated flat retinal tear and avulsed operculum. Posterior hyaloid membrane (large open arrow), operculum (asterisk), laminocytes (small closed arrow). the latter being used to highlight the vitreous gel. In the second group, the globe was placed prone and a $13 \mathrm{~mm}$ trephine centred on the optic nerve was used to remove a disc of posterior sclera, choroid and retina. This approach was chosen to provide access to the posterior aspect of the detached PHM and Weiss' ring which settles under gravity to the anterior vitreous cavity. Using the operating microscope and gentle aspiration of the retrohyaloid fluid, we were then able to resect 'en bloc' whole sheets of the PHM including the Weiss ring from where it had gravitated deep in the anterior vitreous cavity. Specimens were placed directly onto poly-L lysine-coated slides and air dried prior to staining with haematoxylin and eosin or immunohistochemical stains as reported previously.

Samples for scanning electron microscopy (SEM) were fixed in a primary fixative consisting of $4 \%$ glutaraldehyde in $0.1 \mathrm{M}$ phosphate buffer for $24 \mathrm{~h}$. After washing in buffer the samples were placed in a secondary fixative of $1 \%$ osmium tetroxide in buffer for $2 \mathrm{~h}$, buffer washed and then dehydrated in an ascending series of alcohols to $100 \%$. Specimens were then Critical Point Dried employing a Polaron E 3000 CPD using fresh dry 100\% alcohol (dried over molecular sieve) and then dried using liquid $\mathrm{CO}_{2}$. Specimens were mounted onto SEM stubs, sputter coated with AuPd using a Polaron E5000 Sputter Coater (approximate thickness $15 \mathrm{nM}$ ). Viewing was with a Philips XL 30 FEG microscope.

The specimens for transmission electron microscopy (TEM) were fixed in $2.5 \%$ glutaraldehyde in $0.1 \mathrm{M}$ phosphate buffer $\mathrm{pH} 7.2$ for $30 \mathrm{~min}$, washed in buffer, post-fixed in $1 \%$ osmium tetroxide, washed in distilled
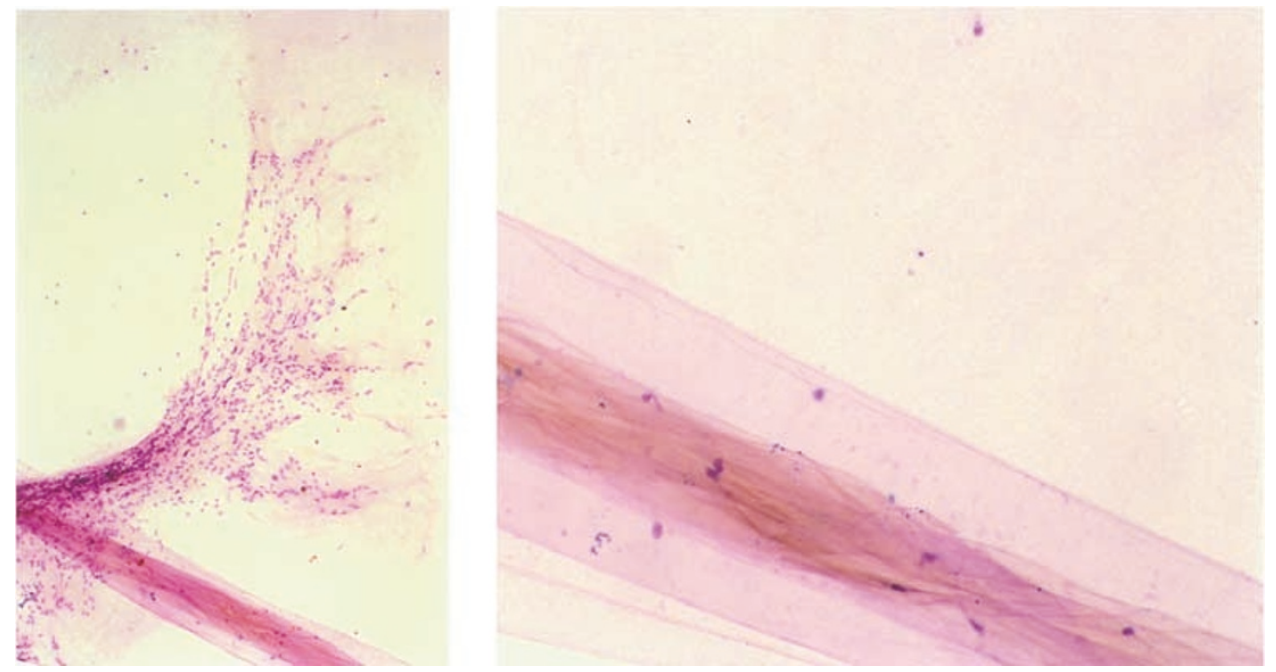

Figure 5 A Weiss' ring and surrounding PHM have been resected 'en bloc' and flat mounted on poly-L lysine-coated slide and air dried, before staining with H\&E. Low power (left) shows dense laminocyte array around Weiss' ring. The cellular density diminishes peripherally (right). 
water, en bloc stained with $2 \%$ aqueous uranyl acetate, dehydrated through an ascending series of alcohols to $100 \%$, immersed in two changes of propylene oxide, 50:50 propylene oxide to resin (overnight) and finally embedded in resin. Ultrathin sections were cut on an LKB ultratome III, stained with uranyl acetate and lead citrate and examined with a Philips C100 electron microscope.

\section{Results}

Eighty-five patients were examined with ages ranging from 68 to 98 yrs (mean 83.4 yrs). The posterior hyaloid membrane had clearly separated from the retina in $66 \%$ of eyes. Twenty-nine eyes from 15 patients have so far been examined by light or electron microscopy.

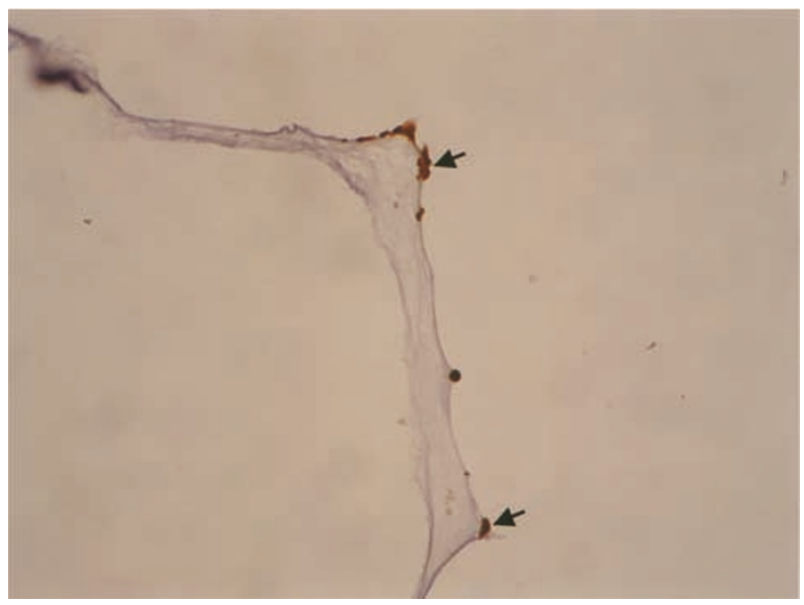

Figure 6 Detached posterior hyaloid membrane showing focal positivity for GFAP (arrow) $\times 400$.

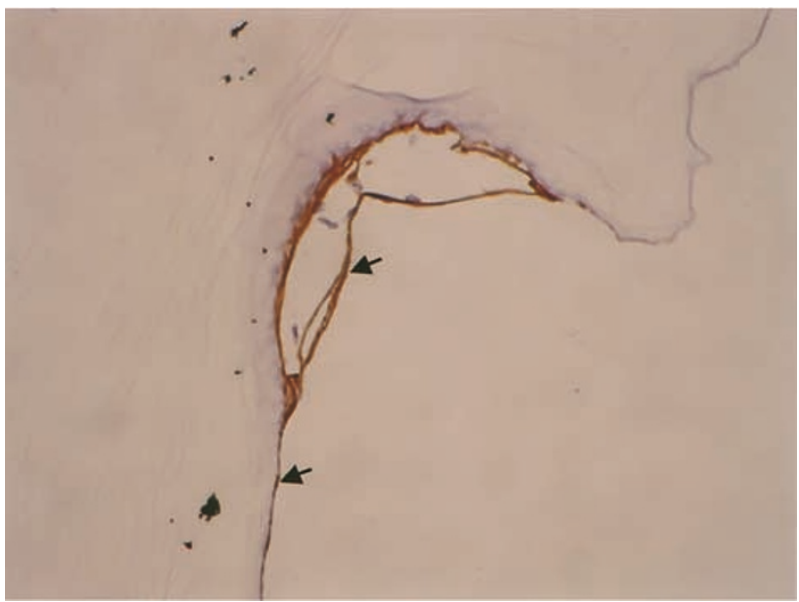

Figure 7 Detached posterior hyaloid membrane. Positive stain for type 4 collagen within the PHM (arrow) $\times 400$.

\section{Light microscopy}

Those eyes with clinical detachment of the posterior hyaloid membrane exhibited a cellular component, most densely populated around the Weiss' ring (Figure 5), which became less densely populated away from the posterior pole (Figures 3, 4 and 5). The cells had oval or round nuclei, indistinct cytoplasm and were only seen within, or abutting, the weakly eosinophilic posterior hyaloid membrane. The posterior aspect of the posterior hyaloid membrane showed a convoluted appearance staining lightly with haematoxylin and eosin. In some cases the Weiss ring showed pigmentladen cells and occasional inflammatory cells in addition to the spindle cells of the membrane itself These additional cell components were absent away from the ring margin, where the morphology of the

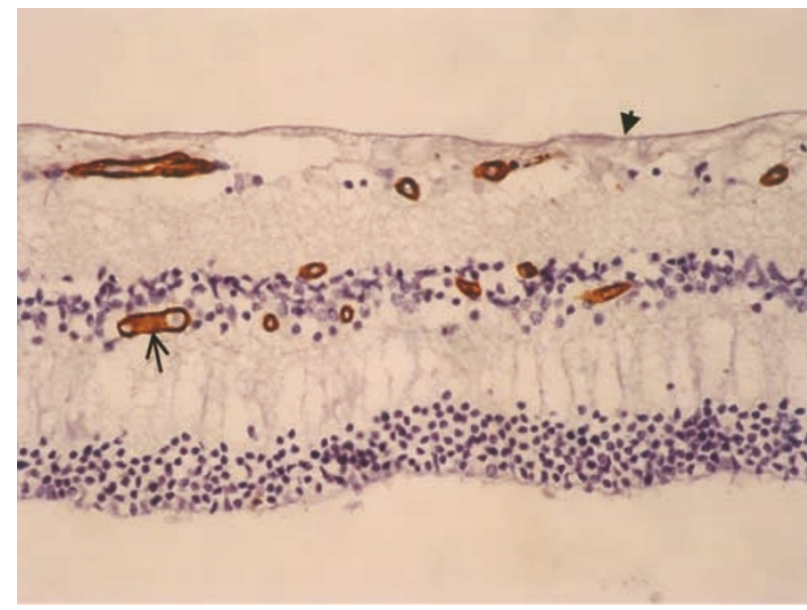

Figure 8 Attached vitreous showing variability of intact ILM stain for type IV collagen (small closed arrow). Note positive stain on retinal vessels as internal control (large open arrow) $\times 400$

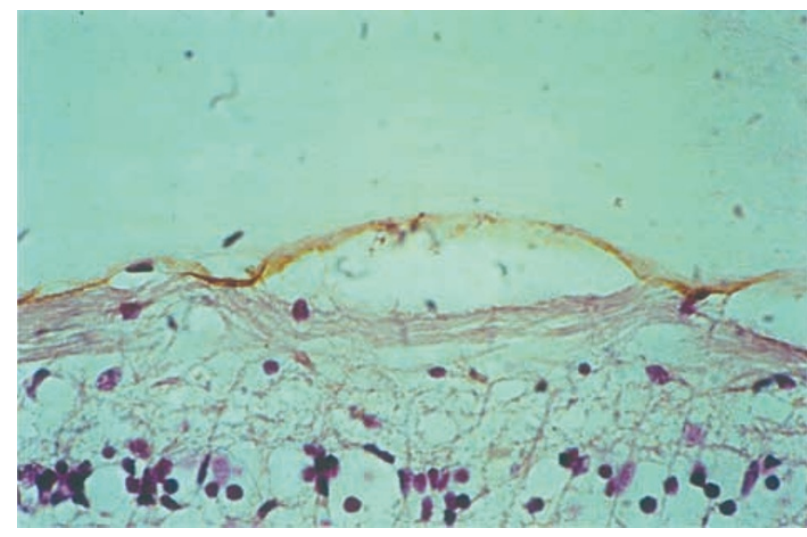

Figure 9 Clinically antemortem attached posterior hyaloid membrane: 80-yr-old patient, high myopia. Stain for type IV collagen $(\times 400)$. Compare with Figure 14a and $b$. 
membrane appeared remarkably uniform and contained only the pale staining spindle-shaped cells.

\section{Immunohistochemistry}

The detached posterior hyaloid membrane exhibited focal positivity for glial fibrillary acidic protein (GFAP) (Figure 6) and type IV collagen (Figure 7), but this uptake was highly variable. Most exhibited a smudge of positivity for type 2 collagen on vitreous aspect of the PHM and a focal positive stain for type 4 collagen within it, presumably corresponding to the basement membrane seen on EM (see below).

Similarly, the retinal surface behind the detached vitreous showed weak positivity for type IV collagen in one case, but negative staining in the remaining three, despite strong positive staining of the retinal vasculature providing an internal control.

In those eyes with clinically attached gel there was focal weak positive type IV stain which was again highly variable. In some cases the ILM stained negatively for type IV collagen but with positive staining of the retinal vasculature providing an internal control (Figure 8), in others the type IV stain was positive as expected (Figure 9). In one case with attached gel there was artefactual splitting of the ILM with focal positivity for GFAP. In the remaining case there was GFAP positivity as expected, but no artefactual ILM split.

\section{Electron microscopy}

Low power scanning images demonstrate the structural contrast between the posterior hyaloid membrane and Weiss' ring seen clinically and its adjacent cortical gel. The cellular component appears sequestered on the

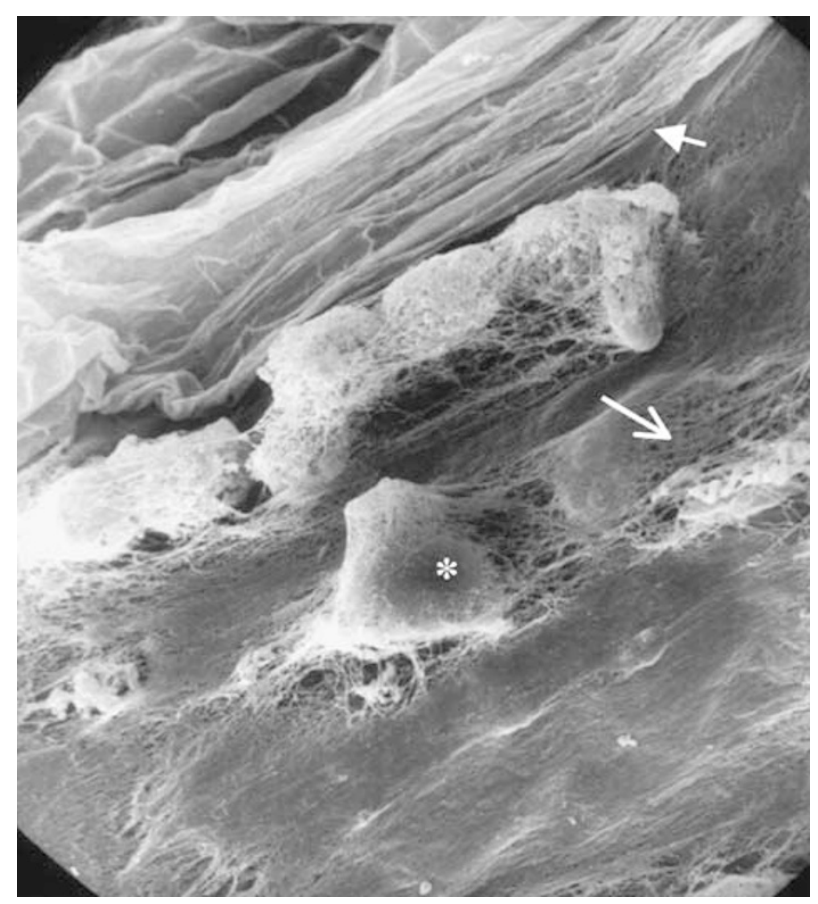

Figure 11 Posterior hyaloid membrane. High power scanning electron micrograph showing contrast between PHM (small arrow) and cortical vitreous (large arrow). *Laminocyte.

vitreous aspect of the PHM, or within the membrane itself (Figures 10 and 11). This can also be demonstrated in cross-section by transmission images as shown in Figure 12. In this image the convoluted nature of the 'free' PHM is demonstrated. The membrane has however retained the association with collagen fibres, a distinct region being visible on one side of the membrane. The basement membrane (Figure 13) is in association with its cellular
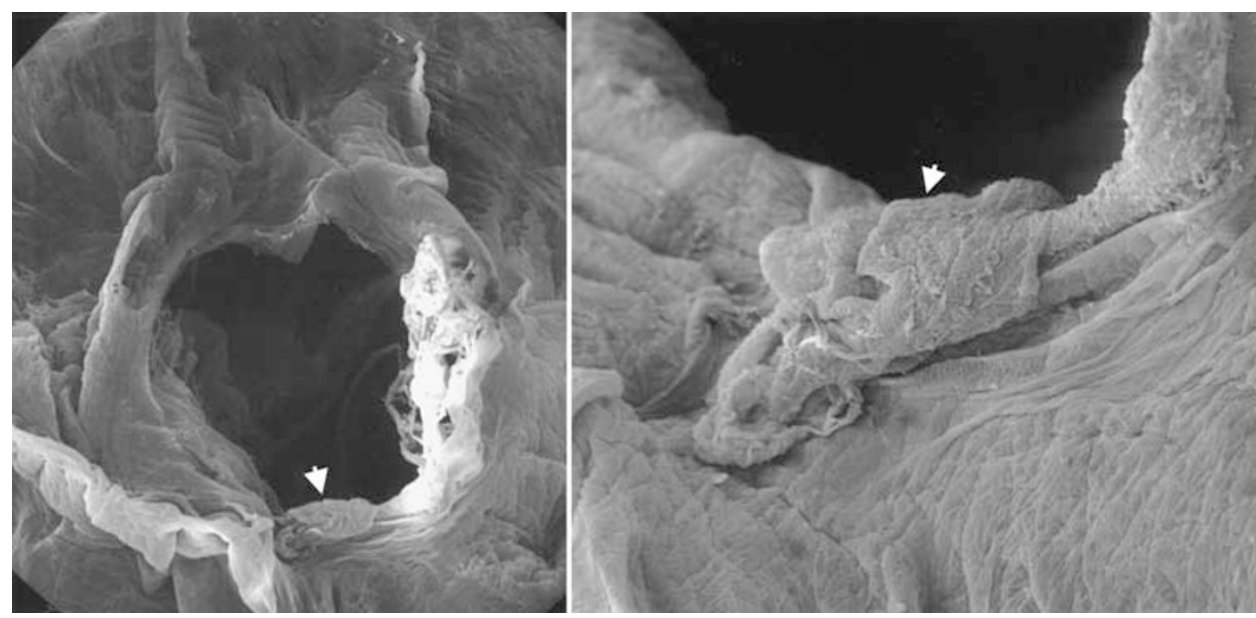

Figure 10 Weiss' ring. Low power scanning electron micrograph of the posterior aspect of a detached Weiss ring. Note uniform appearance and cortical gel spill-over into retrohyaloid space (arrow) $\times 10$. 


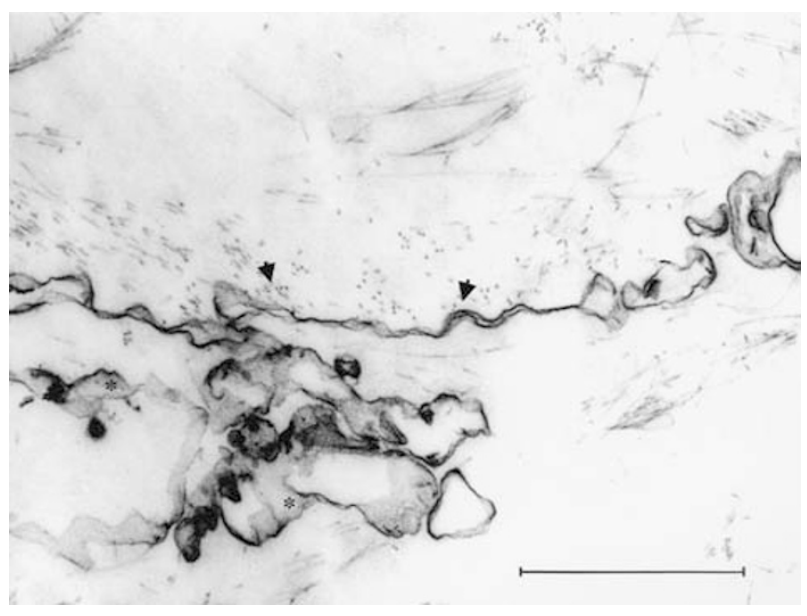

Figure 12 Transmission electron micrograph showing extensive convoluted PHM. In some areas (*) the membrane is shown in tangential section. Collagen fibres, LS and TS (arrows) can be seen to be more heavily associated with one side of the membrane but are present throughout the gel. Scale bar $=1 \mu \mathrm{m}$.

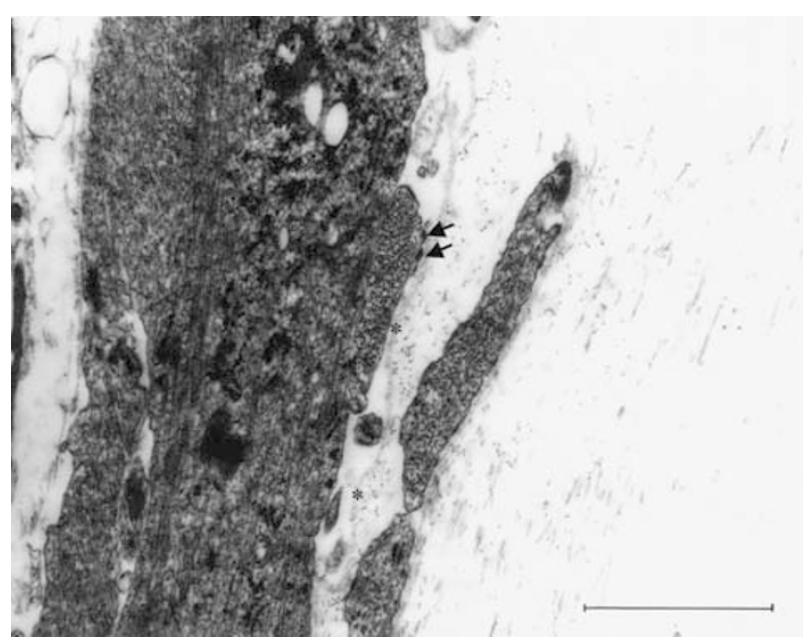

Figure 13 High power TEM demonstrates both fibres and BM $\left.{ }^{*}\right)$ associated with the cellular component of the specimens from patients with clinically detached PHM. Hemi-desmosome attachment plaques (arrows) can be seen between the cells and $\mathrm{BM}$. In places the BM appears to be losing its association with the underlying cells. Scale bar $=1 \mu \mathrm{m}$.

component, and attachment plaques of hemidesmosomes are present.

\section{Summary}

The structural and cellular arrangements of the posterior hyaloid membrane and its relationship with vitreous detachment and the inner limiting membrane were reported by Straatsma and Zimmerman almost 40 years ago. ${ }^{1}$

This study attempts to extrapolate this work by correlating the immunohistochemistry and ultrastructural features of the posterior hyaloid membrane, with known antemortem findings. It illustrates the contrast between the complex cellular arrangement within the PHM and the adjacent cortical gel. The structural and cellular arrangements illustrated here confirm those previously reported ${ }^{1}$ and illustrated in Figures 14a and 14b (reproduced with permission).

The type IV collagen antibody used in this study recognises all basement membranes except corneal basement membrane, and Descemet's (type VIII collagen). There are no data available for ILM but clearly there is variability of uptake of stain in control eyes with both attached and detached vitreous (Figures 8 and 9). This variability might be due to a variety of factors including:

(i) Poor recognition of ILM basement membrane.

(ii) Degenerative changes in ILM/PHM over a longer time scale.

(iii) Problems with antigen retrieval of globes after prolonged fixation and longer processing and variety of clearing agents, eg changing from chloroform to xylene.

It is likely that the cellular component within the PHM will influence a wide variety of vitreoretinal disorders particularly cellophane maculopathy, macular pucker, macular hole and perhaps PVD itself. The introduction of the term hyalocyte is attributed to Balazs $^{10}$ and a possible glial cell origin has been suggested. The hyalocyte as a cell type remains to be accurately categorised, and the term is currently used for any non-inflammatory cell believed to normally reside in the vitreous.

In this study we have illustrated the presence of cells intimately associated with the posterior hyaloid membrane/inner limiting membrane, and absent from the rest of the vitreous. The evidence presented here: presence in eyes with and without PVD, secretion of fibrillary and type IV collagen, presence of hemidesmosomal junctions, suggests these cells have a function in maintaining the PHM/ILM.

As a result of this study we propose the cellular component of the PHM to be designated laminocytes in order to emphasise their type IV collagen and basement membrane association and planar array. Further studies on the cellular changes within the PHM will be important to explore the pathological changes associated with vitreoretinal disease.

\section{Acknowledgements}

The authors gratefully acknowledge the help of patients and relatives who have assisted in this study. 
a

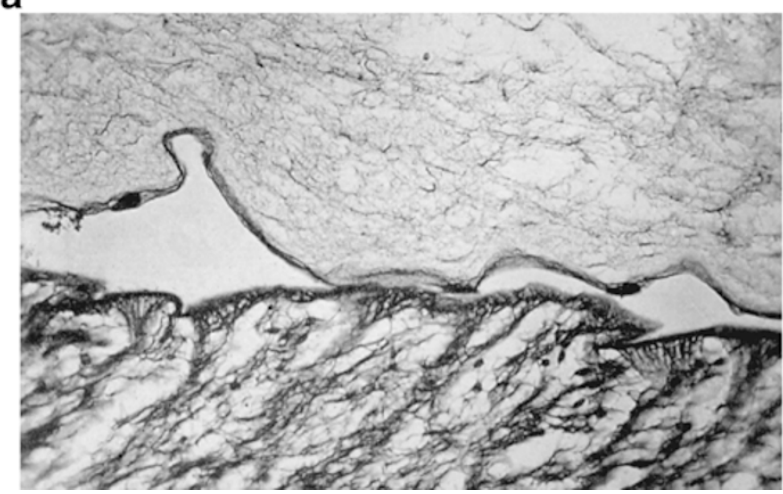

b

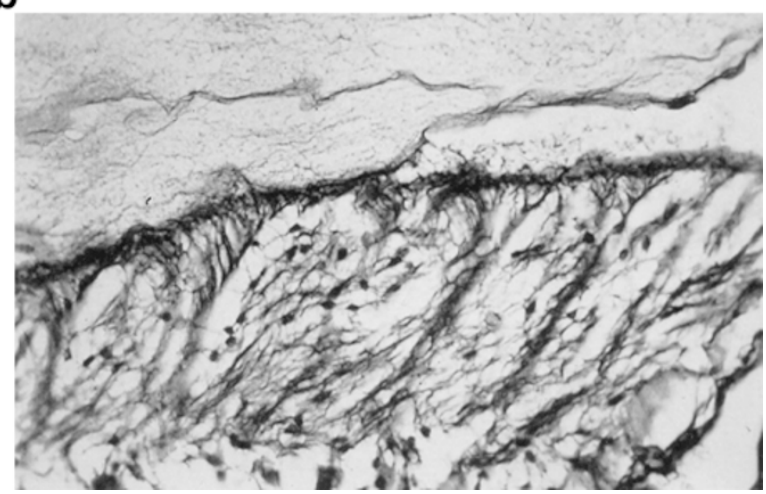

Figure $14(\mathrm{a}-\mathrm{b})$ Anatomic relationships of the retina to the vitreous body and to the pigment epithelium. Reproduced with kind permission of Dr Bradley Straatsma. ${ }^{1}$ Note spindle-shaped cells integral to the separated posterior hyaloid membrane.

We also thank Mr Philip Ball and the medical illustration Department at Addenbrooke's Hospital. The University of Cambridge Retinal Research Fund, The London Law Trust and The Isaac Newton Trust supported this work.

\section{References}

1 Zimmerman LE, Straatsma BR. Anatomic relationships of the retina to the vitreous body and to the pigment epithelium. In: Schepens CL (ed). Importance of the Vitreous Body in Retina Surgery with Special Emphasis on Reoperations. CV Mosby Co: St Louis, 1960, pp 15-28.

2 Gärtner J. Electron microscope observations of the relationships between vitreous body and retina. Mod Prob Ophthalmol 1966; 4: 67-75.

3 Foos RY. Posterior vitreous detachment. Trans Am Acad Ophthalmol Otolaryngol 1972; 76: 480.
4 Foos RY. Vitreoretinal juncture; topographical variations. Invest Ophthalmol 1972; 11: 801-808.

5 Heegaard S, Jensen OA, Prause JU. Structure and composition of the inner limiting membrane of the retina. Graefe's Arch Clin Exp Ophthalmol 1986; 224: 355-360.

6 Heegaard S. Structure of the human vitreoretinal border region. Ophthalmolgica 1994; 208: 82-91.

7 Seebag J. Anatomy and pathology of the vitreo-retinal interface. Eye 1992; 6: 541-552.

8 Snead MP, Snead DRJ, Mahmood A, Scott JD. Vitreous detachment and the posterior hyaloid membrane: a clinicopathological study. Eye 1994; 8: 204-209.

9 Snead MP, Snead DRJ, Harrison JB, Scott JD. Vitreous detachment and the posterior hyaloid membrane: a clinical, histological and ultrastructural study. Inv Ophthalmol Vis Sci 1997; A3160.

10 Balazs EA. The vitreous. In: Davson H (ed). The Eye. London Academic Press, Vol 1a, 1984, pp 533-589. 